\title{
Praxis educativa constructivista como generadora de Aprendizaje Significativo en el área de Matemática
}

\section{Constructivist educational praxis as a generator of Significant Learning in the Mathematics area}

\author{
Yanedis Ruth Miranda-Nuñez \\ janedis7691@gmail.com \\ Institución Educativa San José Nro 2, Magangué \\ Colombia \\ https://orcid.org/0000-0002-5596-5729
}

Recibido: 1 de octubre de 2019

Aprobado: 12 de diciembre de 2019

\begin{abstract}
RESUMEN
Enseñar y aprender matemáticas implica aproximarse a los números y las formas que, en tanto objetos lógicos e ideales, exigen, por una parte, una praxis educativa orientada a enriquecer sus posibilidades de utilización y, por la otra, considerar que el conocimiento es una construcción activa del sujeto que aprende. En tal contexto, la presente investigación se plantea como propósito describir una praxis educativa constructivista como generadora del aprendizaje significativo de las matemáticas. Metodológicamente se desarrolló bajo la metodología del análisis descriptivo documental revisando documentos científicos para identificar y analizar aspectos asociados al tema adaptados a las nuevas exigencias de la sociedad que tiene necesidades de ciudadanos bien formados.
\end{abstract}

Descriptores: Praxis Educativa; constructivista; generadora; aprendizaje Significativo; matemática.

\section{ABSTRACT}

Teaching and learning mathematics implies approaching the numbers and forms that, as logical and ideal objects, require, on the one hand, an educational praxis aimed at enriching its possibilities of use and, on the other, considering that knowledge is a construction active of the learning subject. In this context, this research aims to describe a constructivist educational praxis as a generator of meaningful learning of mathematics. Methodologically, it was developed under the methodology of descriptive documentary analysis, reviewing scientific documents to identify and analyze aspects associated with the subject and adapted to the new demands of society that has well-trained citizens' needs. 
Descriptors: Educational Praxis; constructivist; generator; Significant learning; Math.

\section{INTRODUCCIÓN}

El constructivismo como marco pedagógico y filosófico de la educación implica una concepción del ser humano como constructor de su propio saber, es aceptar que los estudiantes ya poseen ciertos conocimientos sobre los aspectos a tratar y pueden facilitar o dificultar nuevos aprendizajes. Dentro del constructivismo, el aprendizaje significativo consiste en un proceso por medio del cual el estudiante, para aprender, relaciona los conceptos nuevos con los conceptos que posee, así como los conceptos nuevos con la experiencia que tiene. Luego, el trabajo de aula del docente es organizar el espacio, los materiales y guiar al estudiante hacia el logro de su aprendizaje.

En este contexto, enseñar y aprender matemáticas involucra la tríada estudiantemaestro-contenido como pilar sobre el cual se asienta la aproximación a los números y las formas que, en tanto objetos lógicos e ideales, exigen, por una parte, el logro de ese aprendizaje significativo que les permita a los estudiantes desarrollar las competencias necesarias. Por la otra, requieren una praxis educativa orientada a enriquecer sus posibilidades de utilización, considerando que el conocimiento es una construcción activa del sujeto que aprende. Por eso, el trabajo del docente en el aula configura prácticas que sólo pueden ser comprendidas en función de la orientación constructivista o no-, de las concepciones subyacentes, así como del entorno en el que se desarrollan.

Al indagar por la praxis educativa en el área de matemáticas, sus concepciones y la visión del aprendizaje significativo que poseen los estudiantes, se pretende involucrar las reflexiones del docente sobre su quehacer pedagógico, cómo lo hace, cuándo lo hace y por qué lo hace. Esto es así, sobre todo si se tiene en cuenta que su práctica está conformada por un cúmulo de acciones, conocimientos, sentimientos, creencias, valores que, consciente o inconscientemente, despliega en el aula con intenciones educativas y repercuten en el estudiante. 


\section{DESARROLLO TEÓRICO}

\section{Praxis Educativa}

En la acepción general, praxis se entiende como sinónimo de práctica, en el sentido de acción o actuar. Runge y Muñoz (2012 p. 78) realizaron un estudio para discernir si la educación es un saber práctico que lleve a definirla como praxis educativa. Para ello siguen las ideas de Aristóteles y exponen que "La praxis es un hacer humano a partir del cual se ve transformado lo humano mismo. Solo los seres humanos son seres de la praxis ya que en ella y con ella se puede y tiene que decidir o tomar decisiones. La decisión necesaria o por tomar remite o surge entonces de la reflexión (la praxis es un hacer libre, con finalidad en sí mismo y reflexionado)".

Quiere decir que praxis refiere a la acción orientada por ideas, intencional, reflexionada y responsable del ser humano puesto que responde a una necesidad humana. Los autores mencionados explican que una actividad puede ser distinguida como praxis si cumple con dos condiciones: primero, si su origen o su necesidad radican en el carácter imperfecto del ser humano; segundo, si actúa sobre el ser humano de tal modo que determinados cambios sean provocados solo por dicha actividad y no son resultado del carácter imperfecto del ser humano.

De allí deducen que la educación es una praxis: el ser humano necesita ser educado y es mediante la educación que se forma el ciudadano que exige el país. Por su parte, Masi (2008, p. 78), tomando como base el concepto de praxis que emplea Freire, expone que la praxis es "Reflexión y acción como unidad indisoluble, como par constitutivo de la misma y por lo tanto imprescindible. La negación de uno de los elementos del par desvirtúa la praxis, transformándola en activismo o un subjetivismo, siendo cualquiera de los dos una forma errónea de captar la realidad. La tensión entre este par dialéctico es una cuestión que constantemente se repite en toda práctica social".

La autora enfatiza la reflexión como elemento constitutivo de la praxis, independientemente del tipo de práctica de que se trate, social, educativa, entre otras. En consecuencia, se entiende que la educación es una praxis, pero, para develar si la acción del docente en el aula es una praxis en tal sentido, es necesario saber si reflexiona sobre su acción educativa. 
Así, la praxis educativa involucra las reflexiones del docente sobre su quehacer pedagógico, cómo lo hace, cuándo lo hace, por qué lo hace, para qué lo hace. Esto es así, sobre todo si se tiene en cuenta que su práctica está conformada por un cúmulo de acciones, conocimientos, sentimientos, creencias, valores que, consciente 0 inconscientemente, despliega en el aula con intenciones educativas y repercuten en el estudiante. Esta reflexión es de suma importancia porque, para realizar una práctica dentro del constructivismo, debe tener presente que el eje de su praxis es el estudiante, no el contenido ni él mismo como poseedor del conocimiento.

En relación con la actividad del docente y dentro de una concepción constructivista, Contreras y Contreras (2012) plantean que "la práctica pedagógica está directamente relacionada con las distintas actividades que diariamente los educadores realizan en un ambiente de clase, donde el actor principal está representado por los alumnos, guiados intencionalmente por un educador." (p. 209). En ese sentido, la práctica pedagógica constituye una acción a través de la cual los docentes facilitan procesos de aprendizajes e incluye una diversidad de elementos: las estrategias de enseñanza, la comunicación pedagógica, la planificación didáctica, el currículo, alumnos, docentes y saberes, que se vinculan para hacer de la educación un proceso continuo, que contribuye con la formación integral de la personalidad de cada individuo.

La praxis educativa para Romero, Tobos, Jinete y Lindo (2006) es la cristalización en modalidades de actividades y éstas en tipos de actos, de carácter educativo. Por eso quienes pretendan investigar la praxis de ciertos actores sociales institucionales tienen que partir de su cristalización en actos-actividades. En el caso de la educación, la praxis se presenta como una variedad y variación de actos, como los actos pedagógicos, la investigación formativa o investigación en general, la extensión, actos administrativos (toma de decisiones y tareas de gestión), actos de bienestar estamental (lúdicos como los artísticos, recreacionales y deportivos y de salud física y mental) y actos de trabajo social.

La praxis educativa, entonces, debe considerar todos los elementos que pueden conducir a un proceso educativo. Es decir, que las prácticas deben suponer 
innovación constante, creatividad, expansión de la imaginación, desarrollo del pensamiento, intercambio de ideas, perfeccionamiento docente académico, de estrategias, de talleres, de momentos de reflexión, de consenso de proyectos, de puntos de vistas, de acercamiento a la realidad, de propuestas visionarias con salida laboral para aquel que no quiere o no puede seguir dentro del sistema. Se trata de entregar herramientas para todos, sea cual sea su propósito personal o social a seguir, proponer prácticas educativas auténticas ya que cada uno de nosotros debe ser protagonistas de nuestra práctica. (Freire, 1999)

La práctica educativa desde la posición de García (2009) es un producto de una compleja articulación entre las teorías vulgares y las científicas, entre el saber, el enseñar y el aprender, entre el conocimiento sabio y el conocimiento artesanal, poniendo en juego estrategias, contenidos, teorías y prácticas sistemáticas como también asistemáticas.

Asimismo, Martos y Martos (2014 p.129) aluden a los distintos elementos que incorpora la praxis educativa al señalar que "las prácticas y los artefactos educativos no pueden ser entendidos fuera de estas distintas mentalidades y contextos, pues si lo que a un profesor le interesa es enseñar a pensar, a aprender o a crear, las prácticas letradas serán radicalmente distintas a las de una enseñanza expositiva tradicional".

En síntesis, vemos que la práctica educativa del docente incorpora una gran cantidad de elementos y acciones: lograr el aprendizaje significativo de los estudiantes, enseñar, estrategias de enseñanza constructivista, estrategias de aprendizaje, entre otros. La importancia del docente y su acción educativa para el logro de un aprendizaje significativo se puede observar en diferentes autores.

Aquí, cabe mencionar a Ausubel y otros (2000, p. 96), quienes señalan "El aprendizaje significativo es aquel aprendizaje en el que los docentes crean un entorno de instrucción en el que los alumnos entienden lo que están aprendiendo, es el que conduce a la transferencia y sirve para utilizar lo aprendido en nuevas situaciones, en un contexto diferente, por lo que más que memorizar hay que comprender, este aprendizaje se opone al aprendizaje mecanicista".

Del mismo modo, Ballester (2002) expresa que el aprendizaje es significativo cuando 
se conecta la estrategia didáctica del docente con las ideas previas del alumno y se logra presentar la información de manera coherente y no arbitraria, es decir de forma constructiva los conceptos, interconectando los unos con los otros en forma de red del conocimiento.

Por lo cual se debe propiciar el manejo de relaciones no arbitrarias entre los conocimientos previos y los nuevos, puesto que el alumno aprende y depende de sus relaciones anteriores y que exista una disposición subjetiva, una actitud favorable para el aprendizaje por parte del alumno, en función de considerar la motivación como un efecto, pero de igual forma una causa del aprendizaje.

Por otra parte, y ya en relación con la enseñanza de las matemáticas para un aprendizaje significativo, Ruíz (2000), Albarracín y otros (2018), al partir de una concepción de las matemáticas como construcción social, apuestan por una enseñanza dinámica en la que se replantean constantemente tanto los contenidos, como las maneras de comunicarlos. Estudiantes y profesores podrán ver las matemáticas como una actividad del hombre, con vínculos con el arte, la historia, la filosofía y otros campos del conocimiento. Una disciplina en la que también tienen lugar el error, el fracaso y, por supuesto, la creatividad.

Lo antes expuesto, nos conduce a argumentar que el docente, durante su praxis educativa, debe realizar una enseñanza constructivista de las matemáticas y aplicar estrategias adecuadas en el aula, con la finalidad de estimular en los estudiantes un aprendizaje que tenga significado en su desarrollo cognitivo y en el mundo material que lo rodea.

\section{Constructivismo}

El auge de tecnología durante la segunda mitad del siglo XX influye en todos los ámbitos de acción del ser humano y trae consigo la aparición de las teorías cognitivas de aprendizaje, las cuales surgen como una alternativa al predominio del conductismo en el proceso de enseñanza y aprendizaje. Estas teorías tienen como base el modelo de procesamiento de información, por lo que enfatizan la racionalidad del hombre, asumiendo que éste posee sistemas perceptuales, sistemas motores y de respuesta, sistemas de memoria y sistemas para el 
razonamiento y la deducción, sistemas que le permiten conocer/aprender.

No obstante, el ser humano no es sólo intelecto puro capaz de un diálogo lógico, de percibir, recordar/olvidar, pensar, razonar y resolver problemas. Se trata de un organismo biológico y está dotado de emociones, conciencia, sistemas de creencias, destrezas, capacidad de supervivencia, entre otras características que no considera el enfoque del procesamiento de información o cognoscitivo, por lo cual este enfoque, tomado aisladamente, resulta insuficiente, inadecuado y engañoso.

De allí que surgen nuevas perspectivas sobre los modos de adquirir conocimientos que incluyen los aspectos humanos obviados en las teorías cognoscitivas. Estas perspectivas se agrupan bajo el concepto de constructivismo, al interior del cual y pese a la diversidad de planteamientos, los autores incluidos coinciden en asumir a los seres humanos como constructores de conocimiento y cultura, capaces de reflexionar sobre sí mismos. En otras palabras: el ser humano es un sujeto cognoscente activo, por tanto, no recibe el conocimiento pasivamente de su entorno o de otros seres humanos.

Se entiende, entonces que no existe una única teoría constructivista, de manera que el constructivismo se puede definir como el conjunto de teorías psicológicas que conciben el conocimiento como una construcción activa del sujeto resultando de su interacción con el ambiente, con los demás y consigo mismo. Esta definición amplia, que incluye una diversidad de variantes según el centro de atención, hace posible hablar de tres tipos de constructivismo: el filosófico, el psicológico y el educativo.

El constructivismo filosófico consiste en una posición epistemológica sobre cómo el ser humano conoce y se asume como una construcción subjetiva. El constructivismo psicológico involucra un conjunto de teorías evolutivas o cognitivas sobre la adquisición/construcción de conocimientos por el ser humano. $\mathrm{Y}$, el constructivismo educativo implica una concepción de la enseñanza y del aprendizaje que se centra en el estudiante como constructor activo de significados y conocimientos sobre los contenidos escolares.

Ya que el presente se refiere a la educación, se centra en el constructivismo educativo. Las definiciones que siguen permiten visualizar los aspectos comunes que poseen y sus visiones sobre la enseñanza y el aprendizaje. Así, según Pozo 
(1996, p. 60), "el conocimiento es siempre una interacción entre la nueva información que se nos presenta y lo que ya sabíamos y, aprender es construir modelos para interpretar la información que recibimos".

Para Díaz y Hernández (2010, p. 25), el constructivismo es "un movimiento pedagógico que considera al aprendiz como un ente activo, capaz de construir su conocimiento sobre la base de sus potencialidades y experiencias, en conjunción con el contexto ambiental que lo rodea".

Por su parte, luego de haber analizado diversas definiciones de constructivismo, Coll y Martin (1997 p.15), lo definen como "un determinado enfoque o paradigma explicativo del psiquismo humano que subraya la importancia de la actividad mental constructiva de las personas en los procesos de adquisición de conocimientos y que, proyectado al ámbito de la educación, conduce a una visión del aprendizaje escolar como un proceso de construcción que el alumnado lleva a cabo a partir de sus conocimientos y experiencias previas y la enseñanza como ayuda a este proceso de construcción".

En este orden de ideas y según los autores ya mencionados, se infiere que, sin prescribir una metodología específica, hace énfasis en que el estudiante es quien construye activamente significados y conocimientos sobre los contenidos escolares; aquí, la función del docente es de guía, orientación y ayuda pedagógica. En consecuencia y entre otros aspectos e implicaciones del constructivismo en los procesos de enseñanza y aprendizaje, cabe mencionar los más importantes para este estudio:

Reconoce la existencia de esquemas o marcos conceptuales o de referencia que posee el estudiante y con los cuales se enfrenta al proceso de enseñanza aprendizaje.

Entiende y acepta la relevancia del solapamiento de lo afectivo con lo cognitivo. En consecuencia, trata de partir de los intereses y motivaciones del estudiante.

Considera la formación de conceptos en interacción con el ambiente, o sea, acepta y asume la experiencia del estudiante, pues, éste en su actividad está acompañado por otros, se relaciona con los objetos del aprendizaje y de la cultura y es capaz de transformarlos. De esta manera reestructura sus conocimientos o 
construye nuevos.

Toma en cuenta la diversidad pues los sujetos que se incorporan al proceso educativo tienen distintos marcos de referencia y diferentes estructuras cognoscitivas. En consecuencia, se necesita enseñar y aprender con modelos y estrategias de aprendizaje variadas, considerando tanto la complejidad del estudiante como la del ambiente donde se desenvuelve.

De acuerdo a Ortiz (2005), se puede pensar en tales aspectos como una interacción dialéctica entre los conocimientos del docente y los del estudiante, que entran en discusión, oposición y diálogo, para llevar a una síntesis productiva y significativa: el aprendizaje. Sin embargo, hay que recordar que éste y la forma en que se realice, aun cuando sean constructivistas, están determinadas por un contexto específico que influye en ambos participantes: docente y estudiantes, debido a sus condiciones biológicas,

Por su parte, para Castillo (2006), el constructivismo sostiene un aprendizaje asentado en unos principios básicos que permiten tomar decisiones sobre el proceso de formación del alumno. Estos principios son:

Principio de racionalidad: logra la reflexión que permite conocer si el proceso de formación está alcanzando el aprendizaje.

Principio de responsabilidad: este principio está implícito en un compromiso con la tarea que se lleva a cabo; tanto en lo que se hace como en la forma en que se hace lo cual implica un nivel de responsabilidad social

Principio de colegialidad: la formación no se hace de forma aislada, depende de un grupo de personas integradas en un órgano colegiado, donde la evaluación oportuna aporta información pertinente al grupo, con la finalidad de que pueda tomar las decisiones necesarias para mejorar el proceso educativo y cumplir la misión para la cual están reunidos.

Principio de profesionalidad: permite mejorar la práctica profesional, ya que el interés se manifiesta en la formación que las personas están recibiendo, pero también por los niveles de profesionalismo demostrados por los docentes en sus tareas.

Principio de perfectibilidad: con base a las debilidades de un proceso formativo está sujeto a cambios continuos que intentan mejorarlo y, así, proporcionar mejores 


\section{Yanedis Ruth Miranda-Nuñez}

condiciones para la formación de buenos profesionales.

Principio de ejemplaridad: la formación profesional otorgada por una institución puede servir de referente a otras formaciones, donde la calidad se aprecia en los resultaos que se aprecian en las respuestas de los estudiantes dentro de un proceso constructivo.

Al reflexionar sobre las implicaciones y los principios del constructivismo antes referidos es evidente que involucran varias teorías psicológicas del aprendizaje. Tomando esto en consideración, se puede afirmar que el constructivismo se trata de un movimiento pedagógico que abarca, en resumen, tres visiones distintas (Díaz y Hernández, 2010): constructivismo psicogenético (representado por Piaget) y referido a la auto estructuración y desarrollo intelectual; el constructivismo cognitivo (Ausubel, Bruner), relacionado con las habilidades del pensamiento y procesamiento de la información y, el constructivismo sociocultural (Vygotski y también Bandura) que hace énfasis en cómo el individuo aprende por medio de la cooperación y su desenvolvimiento social.

Cabe destacar que, no obstante compartir la idea de que el ser humano es un ente activo durante el aprendizaje, ninguno de ellos se denominó como constructivista. La teoría psicogenética del aprendizaje de Piaget se centra en la equilibración de los procesos de asimilación y acomodación. En la asimilación se integran elementos del exterior a estructuras que están en evolución o ya acabadas en el organismo. En ese sentido, "la asimilación sería el proceso por el que el sujeto interpreta la información que proviene del medio, en función de sus esquemas o estructuras conceptuales disponibles" (Pozo, 1997, p. 178).

La acomodación es un proceso complementario que consiste en la modificación de las estructuras cognoscitivas al asimilar nueva información. Pozo (1997 p. 180) expresa que la acomodación explica la tendencia de nuestros conocimientos o esquemas de asimilación para adecuarse a la realidad, así como el cambio de esos esquemas cuando la adecuación no se efectúa. La acomodación supone "una nueva reinterpretación de los datos o conocimientos anteriores en función de los nuevos esquemas construidos".

Así, ambos procesos se implican necesariamente, de tal manera que el progreso de 


\section{Yanedis Ruth Miranda-Nuñez}

las estructuras cognitivas tiene como soporte un equilibrio entre ellos. Se entiende, entonces, que aprender es acomodar, es la adecuación a la realidad, es atribuir significado al mundo como efecto de la organización de las estructuras cognitivas generales de un individuo.

Por otro lado, Pozo (1997 p. 194), cuando Vygotsky toma como base el concepto de actividad, piensa que el ser humano no solo responde a los estímulos, sino que actúa sobre ellos y los transforma, lo que es posible por la mediación de instrumentos que se interponen entre el estímulo y la respuesta. Así, "la actividad es un proceso de transformación del medio a través del uso de instrumentos".

Los mediadores son instrumentos que transforman la realidad, no la imitan; su función consiste en modificar activamente las condiciones ambientales. En una concepción socio-cultural del aprendizaje, para Vygotski (1979), la cultura proporciona los instrumentos necesarios para que el hombre modifique su entorno, adaptándose de manera activa a él. Estos instrumentos pueden ser de dos clases. El más simple es cualquier herramienta que actúa materialmente sobre el estímulo, modificándolo. Como ejemplo se tiene un martillo que actúa directamente sobre el clavo. El segundo tipo de instrumento mediador es de diferente naturaleza y produce una actividad adaptativa distinta, es el signo.

Dado que la cultura está constituida por artefactos, instituciones, valores y símbolos, son los símbolos los que median en nuestras acciones; de ellos, el más usado es el lenguaje. El signo no modifica materialmente el estímulo sino a la persona que lo usa como mediador; básicamente actúa sobre la interacción de la persona con su entorno.

En palabras de Vygotski (1979, p. 91) "la función de la herramienta no es otra que la de servir de conductor de la influencia humana en el objeto de la actividad; se halla externamente orientada y debe acarrear cambios en los objetos. Es un medio a través del cual la actividad humana externa aspira a dominar y triunfar sobre la naturaleza". Por otro lado, el signo no cambia absolutamente nada en el objeto de una operación psicológica. Así pues, se trata de un medio de actividad interna que aspira a dominarse a sí mismo; el signo, por consiguiente, está internamente orientado 


\section{Yanedis Ruth Miranda-Nuñez}

Luego, son los signos que, como mediadores, modifican al sujeto $y$, a través de éste, a los objetos. En el proceso de enseñanza y aprendizaje, los significados provienen del medio social externo y deben ser interiorizados por cada niño concreto. Para Vygotski (1979), el medio social está conformado por objetos y por personas que median en la interacción del niño con los objetos. En esta interacción, el niño -sujeto cognoscente- para conocer/aprender no imita los significados sino que los reconstruye.

En la relación que establece entre desarrollo y aprendizaje, el citado autor distingue dos niveles de desarrollo o dos tipos de conocimiento en las personas: el nivel de desarrollo efectivo y el nivel de desarrollo potencial. El primero representa los mediadores ya internalizados por el sujeto pues se refiere a lo que la persona puede efectuar sin ayuda. El segundo, está constituido por lo que el individuo es capaz de hacer con ayuda de otras personas o de instrumentos mediadores externamente proporcionados.

La diferencia entre ambos la denomina zona de desarrollo potencial. En esta concepción del aprendizaje tienen una especial importancia los procesos de instrucción o enseñanza puesto que constituyen la facilitación externa de mediadores para su internalización; el docente asume la función de mediar, se convierte en mediador.

\section{Aprendizaje Significativo}

Durante mucho tiempo se consideró que el aprendizaje era sinónimo de cambio de conducta debido al dominio de una perspectiva conductista de la labor educativa. Sin embargo, se puede afirmar con certeza que el aprendizaje humano va más allá de un cambio conductual, pues también conduce a un cambio en el significado de la experiencia.

De acuerdo a León (2005), el ser humano tiene la disposición de aprender de verdad sólo aquello a lo que le encuentra lógica. El auténtico aprendizaje es el significativo, el que se aprende con sentido, cualquier otro aprendizaje será puramente mecánico, memorístico, coyuntural, aprendizaje para pasar un examen, para ganar la materia. Según Ausubel, Novak y Hanesian (2000, p. 37) un aprendizaje es significativo 
cuando un contenido nuevo "puede relacionarse, de modo no arbitrario y sustancial (no al pie de la letra) con lo que el alumno ya sabe". La definición implica dos aspectos, el primero plantea que el aprendizaje depende de la estructura cognitiva previa que se relaciona con la nueva información. Debe entenderse por "estructura cognitiva" al conjunto de conceptos e ideas que un individuo posee en un determinado campo de conocimiento, así como su organización (Ausubel y otros, 2000, p. 60).

El segundo aspecto indica que el aprendizaje significativo es relacional, es decir, involucra la capacidad de relacionar que debe poseer el estudiante. Por eso, Díaz y Hernández (2010) explican que el aprendizaje significativo conduce a la creación de estructuras de conocimiento mediante la relación sustantiva entre la nueva información y las ideas previas de los estudiantes. Ese aprendizaje es sistemático, organizado, pues es un fenómeno complejo que no se reduce a simples asociaciones memorísticas, y es aquí donde el estudiante relaciona de manera no arbitraria y sustancial la nueva información con los conocimientos, experiencias previas que ya posee en su estructura cognitiva.

En el mismo sentido, para aprender, el alumno debe estar dispuesto a establecer relaciones sustanciales y no arbitrarias del nuevo material con su estructura cognoscitiva, ya que al aprender codifica, transforma y retiene la nueva información para construir nuevos significados. En ese orden de ideas, Ausubel y otros (2000) distinguen dos tipos de aprendizaje, el memorístico o repetitivo y el significativo, los cuales conforman un continuo.

El aprendizaje por repetición es mecánico, pues involucra la adquisición memorística de conocimientos, sin ningún significado e inaplicable en situaciones y contextos diferentes. Se efectúa en una enseñanza por recepción basada en la instrucción expositiva que comunica el contenido que va a ser aprendido en su forma final.

El aprendizaje significativo implica una construcción de aprendizajes por parte del alumno quien, con la ayuda del profesor, relaciona de forma no arbitraria contenidos nuevos tomando como base los conocimientos previos que posee. Es decir, el docente guía, organiza el material para que el estudiante comprenda y construya nuevos significados con la información. 
Por otra parte, en función de la naturaleza del conocimiento adquirido, los autores mencionados señalan la existencia de tres tipos de aprendizaje significativo: de representaciones, de conceptos y de proposiciones.

El aprendizaje de representaciones es el más elemental y consiste en la atribución de significados a determinados símbolos; se trata fundamentalmente de la adquisición de vocabulario.

El aprendizaje de conceptos, definidos como "objetos, eventos, situaciones o propiedades de que posee atributos de criterios comunes y que se designan mediante algún símbolo o signos" Ausubel y otros (2000, p. 61). Los conceptos se forman a partir de los objetos durante la experiencia y se adquieren a partir de conceptos previos. En la enseñanza es importante el trabajo experimental para un aprendizaje por descubrimiento, ya sea guiado o autónomo.

El aprendizaje de proposiciones, el cual va mas allá de la asimilación de lo que representan las palabras, combinadas o aisladas porque exige captar el significado de las ideas y expresarlas en forma de proposición. Es importante hacer notar que, una proposición es la relación entre varios conceptos por lo que el aprendizaje se adquiere a partir de conceptos preexistentes. Durante la enseñanza, entonces, es de suma importancia tener presentes los conocimientos previos de los estudiantes, en especial la estructura conceptual del área de aprendizaje.

\section{Enseñanza constructivista}

Como eje de la praxis educativa, enseñar es la primordial actividad que realiza el docente. Al respecto, Carvajal y Gómez (2012) señalan que la enseñanza está configurada por representaciones individuales de la realidad válidas y creíbles que permiten guiar el pensamiento y el comportamiento que se forman tempranamente, tienden a permanecer aún ante fuertes contradicciones lógicas y crean un filtro a través del cual los fenómenos son interpretados y la información es procesada por el alumno.

Según Raths y McAninch (2013), la enseñanza se basa en las acciones que el docente plantea, tales como planear, realizar su praxis, reflexionar y evaluar. A su vez, los docentes son influenciados por diversos factores como ciertas políticas 
educativas, macro-estructura escolar, currículo en todos los niveles educativos, sociedad, cultura, familia y economía, entre otros.

Dado que en la sociedad actual los roles del docente en los procesos de enseñanza y aprendizaje han cambiado, ya no puede ser un simple transmisor de información frente a un alumno que solo recibe y memoriza. Según Díaz y Hernández (2010, p.2), se espera que el estudiante "aprenda a aprender, a colaborar, a comportarse de forma ética, responsable y solidaria, a resolver problemas, a pensar y recrear el conocimiento".

En consecuencia, el profesor asume el rol de agente mediador de los procesos que conducen a los estudiantes a construir su conocimiento y a desarrollar las capacidades mencionadas. Para esto, debe no solo dominarlas, sino apropiarse de nuevas formas de enseñar orientadas hacia el constructivismo, en el cual el estudiante es el protagonista. Como lo indican, también, Alvarado y Rodríguez (2011), la enseñanza transita de un modelo tradicionalista a uno más dirigido hacia el aprendizaje centrado en el alumno.

La enseñanza enfocada en el estudiante como un sujeto individual y social a la vez, está inmersa en una praxis educativa constructivista, donde el aprendizaje se puede ver desde una perspectiva más individual y endógena debido a la acción del individuo (Piaget); cognitiva que incorpora la acción docente (Ausubel y otros), o bien, una visión socio-cultural (Vygotski), con énfasis en el influjo de la cultura sobre las interacciones del aprendiz con otros y con el medio social en el que se realiza la experiencia educativa. En todo caso, se pretende que el estudiante logre un aprendizaje que tenga significado, tanto durante su proceso educativo como para su vida cotidiana, individual y social-cultural.

Es por ello que, tal como dice Socas (2011), el docente de matemáticas, para desarrollar una praxis educativa constructivista debe poseer un conocimiento profundo de la disciplina o área que enseña -en este caso las matemáticas-, del currículo que pone en práctica y tener el saber didáctico necesario para organizar los contenidos para la enseñanza, pues ahora es un mediador y no un transmisor de información. Su labor consiste en propiciar las herramientas para que el estudiante logre un aprendizaje significativo de las matemáticas. 
En ese sentido, debe contextualizar el conocimiento que pretende enseñar y secuenciar cada uno de los bloques de contenido de acuerdo con su estructura, así como tomando en cuenta las capacidades cognitivas que los estudiantes poseen y/o pueden desarrollar. Es decir, para realizar una enseñanza constructivista orientada hacia el aprendizaje significativo, el docente debe indagar por el aprendizaje de experiencias, hechos y objetos que ya posee el estudiante (conocimientos previos), ofrecer o crear materiales que faciliten la incorporación sustantiva y no arbitraria de los nuevos conocimientos en la estructura cognitiva de los alumnos; inquirir por los intereses y motivaciones de los estudiantes para implicarlos afectiva y efectivamente en la búsqueda o construcción de relaciones nuevas; motivar al estudiante para que realice el esfuerzo de relacionar los conocimientos y conceptos nuevos con los ya existentes en su estructura cognitiva.

Estas características de la enseñanza constructivista implican el uso de una serie de estrategias docentes o didácticas que configurarán su praxis educativa. Diferentes autores: Díaz y Hernández (2010), González 82012), Albarracín y otros (2018), entre otros, proponen diversas estrategias dirigidas a lograr el aprendizaje significativo del estudiante. Así se tienen: plantear metas claras para encontrar sentido al proceso, contextualizar y relacionar los contenidos de matemáticas con otras áreas del conocimiento y la vida cotidiana, utilizar ilustraciones, organizadores previos, preguntas intercaladas, ejemplos y analogías, mapas conceptuales y redes semánticas, resúmenes, textos seleccionados y otros materiales como películas, canciones, instrumentos, entre otras muchas estrategias.

Con respecto a ello, Albarracín y otros (2018), explican que las estrategias son útiles para crear entornos apropiados que faciliten el poder enseñar matematizando, pero esto requiere situaciones y problemas matemáticos que tengan un contexto significativo para los estudiantes. Se sustenta en una concepción de la matemática como actividad humana accesible a todos que se aprende haciendo. Por eso los problemas presentados deben utilizar herramientas matemáticas para su organización y solución.+ 


\section{METODOLOGÍA}

En el paradigma cualitativo no existe un método único de investigación; al contrario, hay múltiples alternativas metodológicas para conocer lo social, el fenómeno humano y los procesos que ocurren en la sociedad; se trata de una investigación vivida, sentida y expresada. En ese orden de ideas, Taylor y Bogdan (1992) señalan que en la investigación cualitativa se producen datos verdaderamente descriptivos, las propias palabras de las personas, habladas o escritas, así como también la conducta observable. Esto favorece la acción práctica, la interpretación, la comprensión y facilita la redacción, ya que en esta modalidad no se impone reglas rígidas al proceso de investigación, pero, sí la necesaria rigurosidad.

Es por ello que en este apartado, el investigador se nutre con la literatura y crea el fenómeno de estudio, comienza con la disyuntiva del alcance de su investigación. En este sentido, resaltan los estudios descriptivos, los cuales consisten primordialmente en especificar las propiedades importantes de un análisis, sin alterar el factor de estudio.

De allí entonces que pueda señalarse que en todo estudio, los descriptivos tienen ventajas y desventajas, entre las primeras: sirven como puerta de entrada para facilitar la realización de estudios analíticos, útiles para identificar diferentes variables y pueden guardar relación con el fenómeno de estudio por lo que pueden identificar grupos vulnerables.

De manera general, según García (2014) los estudios descriptivos buscan especificar las propiedades importantes de personas, grupos, comunidades 0 cualquier otro fenómeno que sea sometido a análisis, por lo que miden o evalúan diversos aspectos, dimensiones o componentes del fenómeno a investigar, por lo tanto, no intervienen o manipulan el factor de estudio, es decir que se observa lo que ocurre con el fenómeno en estudio en la realidad.

En el mismo sentido, menciona Sellitz (1965), en esta clase de estudios el investigador debe ser capaz de definir qué se va a medir, cómo lograr precisión en esta medición y cómo especificar quienes deben estar incluidos en la medición. En pocas palabras, la investigación descriptiva es la que expone de manera más veraz y directa la realidad de un fenómeno de estudio, por ello es necesario conocer sus 
exigencias conceptuales y operativas para mitigar concepciones erradas sobre su alcance.

\section{REFLEXIONES FINALES}

Partimos del siguiente concepto de estrategias: "Es el paradigma o patrón asumido a través de una serie de actividades que ocurren en un determinado tiempo, proporcionando resultados concretos a nivel personal o grupal".

Un mediador de los aprendizajes inmerso dentro del enfoque constructivista de Vigostky deberá tener presente los tres elementos direccionales: La zona de desarrollo próximo, la interiorización y la formación de conceptos.

Un docente constructivista estará permanentemente en prácticas investigadoras mediante una perspectiva metodológica como lo representa la Investigación-Acción, convirtiéndose en un investigador indeterminista rompiendo el cerco del determinismo y abriendo las compuertas hacia la búsqueda de nuevos conocimientos (no verdades absolutas).

Es necesario resaltar, además, la coherencia existente entre la metodología de la Investigación-Acción y la práctica de mediar; de allí que la manera de ejecutar el acto educativo esté signado por un proceso de planificación, acción, observación, reflexión, transformación y así continuar el ciclo en permanente actitud hacía la mejora de la calidad en la educación y en la construcción de aprendizajes significativos.

El docente constructivista tiene en la transposición didáctica un elemento operacionalizador de la acción docente para lograr la interiorización de aprendizajes y la formación de conceptos a través de la zona de desarrollo próximo. Existen estímulos para el desarrollo del potencial creativo y la promoción del pensamiento innovador a través de métodos creativos.

Se impone, entonces su práctica durante el acto educativo; lo cual permite hablar de un constructivismo creativo. Los espacios educativos: salones de clases, laboratorios, canchas deportivas o cualesquiera otro, son los adecuados para establecer estilos comunicacionales, llenos de expresividad para impedir los bloqueos al desarrollo del potencial creativo y contribuyan con la construcción de 
aprendizajes.

\section{REFERENCIAS CONSULTADAS}

1. Albarracin, LI., Badillo, E., Giménez, J., Vanegas, Y., y Vilella, X. (2018). Aprender a enseñar matemáticas en la educación primaria. Madrid: Síntesis.

2. Ausubel, D.; Novak, J. y Hanesian, H. (2000). Psicología educativa. Un punto de vista cognoscitivo. México: Trillas.

3. Ballester, A. (2002). "La realidad del Aprendizaje Significativo". Cuadernos de pedagogía. Año 15, (277). Caracas

4. Carvajal, E. y Gómez, R. (2002). Concepciones y representaciones de los maestros de secundaria y bachillerato sobre la naturaleza, el aprendizaje y la enseñanza de las ciencias. Revista mexicana de investigación educativa [Revista en línea], 16. Disponible: http://www7.uc.cl/sw_educ/educacion/ grecia/plano/html/pdfs/linea_investigacion/Que_Ciencia_Ensenar_IEC/IEC_09 9.pdf [Consulta: 2019, agosto 10]

5. Castillo, S. (2006) Formación del profesorado en educación superior: Desarrollo curricular y evaluación. Madrid: McGraw-Hill.

6. Coll, C. y Martin, J. (1997). Un Marco Referencial Psicológico para la Educación Escolar. La Concepción Constructivista del Aprendizaje y de la Enseñanza. Madrid. Alianza.

7. Contreras, M. y Contreras, A. F. (2012). Práctica pedagógica: postulados teóricos y fundamentos ontológicos y epistemológicos. Heurística, Revista digital de la Historia de la educación, № 15.

8. Díaz, F. y Hernández, G. (2010). Estrategias Docentes para un Aprendizaje Significativo. Una Interpretación Constructivista. México. Mc. Graw-Hill.

9. Freire, P (1999) Pedagogía de la autonomía. Buenos Aires: Siglo XXI.

10. García, J (2014) Estudios descriptivos. Nure Investigación. Disponible en: http://webpersonal.uma.es/ jmpaez/websci/BLOQUEIII/DocbllI/Estudios\%20d escriptivos.pdf.

11. García, M (2009), Aprendiendo a ser humanos: una antropología de la educación, Pamplona, Ediciones de la Universidad de Granada. 
12.León, J (2005). Aprendizaje Significativo. Documento en línea: http://www.psicopedagogia.com/definicion/aprendizaje\%20significativo.

13. Martos, E. y Martos García, A. E. (2014). Artefactos culturales y alfabetización en la era digital: Discusiones conceptuales y praxis educativa. Teoría educativa, № 26, 1-2014, pp. 119-135. [Revista Digital] Disponible en DOI: http://dx.doi.org/10.14201/teoredu2014261119135

14. Masi, A. (2008). El concepto de praxis en Paulo Freire. En Godotti, M.; Gómez, M. V.; Mafra, J.; Fernández de Alencar, A. (Compiladores) (2008). Paulo Freire. Contribuciones para la pedagogía. CLACSO, Consejo Latinoamericano de Ciencias Sociales, Buenos Aires. Enero 2008. Disponible en: http://bibliotecavirtual.clacso.org.ar/ar/libros/campus/freire/09Masi.pdf.

15. Ortiz, D. (2005). El constructivismo como teoría y método de enseñanza. Sophia, Colección de Filosofía de la Educación. [Revista en línea], 19. Disponible en: http://www.redalyc.org/articulo.oa?id=441846096005 [Consulta: 2018, noviembre 6]

16. Pozo, J. (1996). Teorías cognitivas del aprendizaje. $5^{\underline{a}}$ edición. Madrid: Morata.

17. Raths, J. \& McAninch, A. (2013). Teacher Beliefs and classroom performance: the impact of teacher education. The United States of America: Age publishing.

18. Romero P. H., Tobos M., Jinete Miryan, L. (2006). La praxis profesional del docente en formación: ¿formarlo viviendo el pasado, el presente, o la forma de vida del proyecto de sociedad por construir? Revista Iberoamericana de Educación (ISSN: 1681-5653) No 40/5. Edita Organización de Estados iberoamericanos para la Educación, la ciencia y la Cultura (OEI).

19. Ruiz, A., Alfaro, C. y Gamboa, R. (2004). Aprendizaje de las matemáticas: conceptos, procedimientos, lecciones y resolución de problemas. Revista en línea].. Disponible en: https://dialnet.unirioja.es/servlet/articulo?codigo= 5381202 [Consulta: 2018, noviembre 10]

20. Runge Peña, A. K. y Muñoz Gaviria, D. A. (2012). Pedagogía y praxis (práctica) educativa o educación. De nuevo: una diferencia Necesaria. Revista Latinoamericana de Estudios Educativos (Colombia), vol. 8, núm. 2, juliodiciembre, 2012, pp. 75-96. Disponible en: http://www.redalyc.org/articulo.oa?id=134129257005.

21. Selltiz, C. (1965). Métodos de investigación en las relaciones sociales. Madrid: Ediciones Rialp. 
22. Socas, M. (2011). Aprendizaje y enseñanza de las Matemáticas en Educación Primaria. Buenas prácticas. Educación Siglo XXI, Vol. 29 № 2 · 2011, pp. 199224.

23. Taylor, S. y Bogdan, R. (1992). Introducción a los métodos cualitativos de investigación. Buenos Aires: Piados.

24. Vygotski, L. S. (1979). El desarrollo de los procesos psicológicos superiores. Barcelona: Crítica.

\section{REFERENCES CONSULTED}

1. Albarracin, LI., Badillo, E., Giménez, J., Vanegas, Y., and Vilella, X. (2018). Learn to teach mathematics in primary education. Madrid: Synthesis.

2. Ausubel, D .; Novak, J. and Hanesian, H. (2000). Educational psychology. A cognitive point of view. Mexico: Threshing.

3. Ballester, A. (2002). "The reality of Significant Learning". Notebooks of pedagogy. Year 15, (277). Caracas

4. Carvajal, E. and Gómez, R. (2002). Conceptions and representations of secondary and high school teachers about the nature, learning and teaching of science. Mexican Journal of Educational Research [Online Magazine], 16. Available: http://www7.uc.cl/sw_educ/educacion/grecia / plano / html / pdfs / linea_investigacion / Que_Ciencia_Ensenar_IEC / IEC_099.pdf [Consultation: 2019, August 10]

5. Castillo, S. (2006) Teacher training in higher education: Curriculum development and evaluation. Madrid: McGraw-Hill.

6. Coll, C. and Martin, J. (1997). A Psychological Reference Framework for School Education. The Constructivist Conception of Learning and Teaching. Madrid. Alliance.

7. Contreras, M. and Contreras, A. F. (2012). Pedagogical practice: theoretical postulates and ontological and epistemological foundations. Heuristics, Digital Magazine of the History of Education, No. 15.

8. Díaz, F. and Hernández, G. (2010). Teaching Strategies for Meaningful Learning. A Constructivist Interpretation. Mexico. Mc. Graw-Hill

9. Freire, P (1999) Pedagogy of autonomy. Buenos Aires: 21st century. 
10.García, J (2014) Descriptive studies. Nure Research. Available at: http://webpersonal.uma.es/ jmpaez/websci/BLOQUEII//Docbll//Estudios\%20d escriptivos.pdf.

11. García, M (2009), Learning to be human: an anthropology of education, Pamplona, Editions of the University of Granada.

12.León, J (2005). Significant learning. Online document: http://www.psicopedagogia.com/definicion/aprendizaje\%20significativo.

13. Martos, E. and Martos García, A. E. (2014). Cultural artifacts and literacy in the digital age: Conceptual discussions and educational praxis. Educational theory, No. 26, 1-2014, pp. 119-135. [Digital Magazine] Available at DOI: http://dx.doi.org/10.14201/teoredu2014261119135

14. Masi, A. (2008). The concept of praxis in Paulo Freire. In Godotti, M .; Gómez, M. V .; Mafra, J .; Fernández de Alencar, A. (Compilers) (2008). Paulo Freire Contributions to pedagogy. CLACSO, Latin American Council of Social Sciences, Buenos Aires. January 2008. Available at: http://bibliotecavirtual.clacso.org.ar/ar/libros/campus/freire/09Masi.pdf.

15. Ortiz, D. (2005). Constructivism as a theory and teaching method. Sophia, Collection of Education Philosophy. [Online Magazine], 19. Available at: http://www.redalyc.org/articulo.oa?id=441846096005 [Consultation: 2018, November 6]

16. Well, J. (1996). Cognitive learning theories. 5th edition. Madrid: Morata.

17. Raths, J. \& McAninch, A. (2013). Teacher Beliefs and classroom performance: the impact of teacher education. The United States of America: Age publishing.

18. Romero P. H., Tobos M., Rider Miryan, L. (2006). The professional praxis of the teacher in training: to train him living the past, the present, or the way of life of the society project to be built? Ibero-American Journal of Education (ISSN: 1681-5653) No 40/5. Edits Organization of Ibero-American States for Education, Science and Culture (OEI).

19. Ruiz, A., Alfaro, C. and Gamboa, R. (2004). Learning mathematics: concepts, procedures, lessons and problem solving. Online magazine] ,. Available at: https://dialnet.unirioja.es/servlet/articulo?codigo = 5381202 [Consultation: 2018, November 10]

20. Runge Peña, A. K. and Muñoz Gaviria, D. A. (2012). Pedagogy and praxis (practice) educational or education. Again: a difference Needed. Latin American Journal of Educational Studies (Colombia), vol. 8, no. 2, July- 
CIENCIAMATRIA

Revista Interdisciplinaria de Humanidades, Educación, Ciencia y Tecnología

Año VI. Vol. VI. N¹. Edición Especial. 2020

Hecho el depósito de ley: pp201602FA4721

ISSN-L: 2542-3029; ISSN: 2610-802X

Universidad Nacional Experimental Francisco de Miranda (UNEFM). Santa Ana de Coro. Venezuela

Yanedis Ruth Miranda-Nuñez

December, 2012, $\quad$ pp. $75-96 . \quad$ Available at: http://www.redalyc.org/articulo.oa?id=134129257005.

21. Selltiz, C. (1965). Research methods in social relations. Madrid: Rialp editions.

22. Socas, M. (2011). Learning and teaching of Mathematics in Primary Education. Good practices. Education XXI Century, Vol. 29 n $^{\circ} 2 \cdot 2011$, pp. 199-224.

23. Taylor, S. and Bogdan, R. (1992). Introduction to qualitative research methods. Buenos Aires: Piados.

24. Vygotski, L. S. (1979). The development of higher psychological processes. Barcelona: Criticism.

2020 por los autores. Este artículo es de acceso abierto y distribuido según los términos y condiciones de la licencia Creative Commons Atribución-NoComercial-CompartirIgual 4.0 Internacional (CC BY-NC-SA 4.0) (https://creativecommons.org/licenses/by-nc-sa/4.0/) 